\title{
The phosphorycholine moiety of the filarial nematode immunomodulator ES-62 is responsible for its anti-inflammatory action in arthritis
}

\author{
M M Harnett, ${ }^{1}$ D E Kean, ${ }^{1}$ A Boitelle, ${ }^{2}$ S McGuiness, ${ }^{2}$ T Thalhamer, ${ }^{1}$ C N Steiger, ${ }^{1}$ \\ C Egan, ${ }^{2}$ L Al-Riyami, ${ }^{2} \mathrm{M}$ J Alcocer, ${ }^{3} \mathrm{~K}$ M Houston, ${ }^{2} \mathrm{~J}$ A Gracie, ${ }^{1}$ I B Mclnnes, ${ }^{1}$ \\ W Harnett ${ }^{2}$
}

${ }^{1}$ Division of Immunology, Infection and Inflammation, Glasgow Biomedical Research Centre, University of Glasgow, Glasgow, UK; ${ }^{2}$ Strathclyde Institute of Pharmacy and Biomedical Sciences, University of Strathclyde, Glasgow, UK:

${ }^{3}$ School of Biosciences, University of Nottingham, Sutton Bonington Campus, Loughborough, UK

Correspondence to: Professor W Harnett, Strathclyde Institute of Pharmacy and Biomedical Sciences, University of Strathclyde, Glasgow G4 ONR, UK;w.harnett@strath.ac.uk

Accepted 22 July 2007 Published Online First 17 August 2007

\section{ABSTRACT}

Objective: In countries where parasitic infections are endemic, autoimmune disease is relatively rare, leading to the hypothesis that parasite-derived immunomodulators may protect against its development. Consistent with this, we have previously demonstrated that ES-62, a $62 \mathrm{kDa}$ phosphorylcholine (PC)-containing glycoprotein that is secreted by filarial nematodes, can exert antiinflammatory action in the murine collagen-induced arthritis (CIA) model and human rheumatoid arthritisderived synovial tissue cultures. As a first step to developing ES-62-based drugs, the aim of this study was to determine whether the PC-moiety of ES-62 was responsible for its anti-inflammatory actions.

Methods: We compared the anti-inflammatory activity of a PC-free form of recombinant ES-62 (rES-62) and a synthetic PC-ovalbumin conjugate (OVA-PC) with that of native ES-62 in the CIA model and synovial tissues from patients with rheumatoid arthritis.

Results: The anti-inflammatory actions of ES-62 in CIA appear to be dependent on the PC moiety as indicated by the reduction in severity of disease and also suppression of collagen-specific $T$ helper 1 cytokine production observed when testing OVA-PC, but not rES-62. Interestingly, the anti-inflammatory activity of PC did not correlate with a reduction in anti-collagen IgG2a levels. Also, the ES-62-mediated suppression of interferon- $\gamma$ from human patient tissues could be mimicked by OVA-PC but not rES-62 or ovalbumin.

Conclusions: In countries where filariasis is endemic the reduced detection of inflammatory diseases, such as rheumatoid arthritis may be because of the antiinflammatory action of the PC moieties of ES-62. PC may thus provide the starting point for the development of novel, safe immunomodulatory therapies.

In countries where parasite infections are endemic, autoimmune disease is relatively rare. ${ }^{1}$ It has therefore been suggested that parasite infection, and in particular products derived from helminths, may protect us from the development of autoimmunity. ${ }^{2}$ We have previously identified and characterised ES-62, an anti-inflammatory phosphorylcholine (PC)-containing glycoprotein that is secreted by the rodent filarial nematode Acanthocheilonema viteae. ${ }^{4}$ ES-62 is also produced by filarial nematodes that parasitise humans ${ }^{3}$ and hence may play a role in preventing the massive inflammatory responses (eg, elephantiasis) that the worms are capable of inducing. We therefore hypothesised that ES-62 had the potential to inhibit inflammatory responses arising from aberrant immune responses as seen in autoimmune diseases, such as rheumatoid arthritis (RA). Consistent with this, we have recently shown that ES-62 can delay the onset and reduce the severity of inflammation exhibited in the murine collagen-induced arthritis (CIA) model. ${ }^{5}$ These actions of ES-62 were manifested by a reduction in antigen-specific $\mathrm{T}$ helper (Th) 1-type cytokine production and immunoglobulin levels, with no compensatory increase in Th2 responses. Crucially, ES-62 was also found to suppress CIA severity and progression when administration was delayed until after clinically evident disease onset ${ }^{5}$ and the therapeutic potential of ES-62 in arthritic inflammation was further demonstrated using samples from human RA. ${ }^{5}$

PC has been characterised as an immunomodulatory moiety utilised by a number of pathogens ${ }^{6}$ and consistent with this, several of the immunomodulatory effects of ES-62 are known to be facilitated by the PC moiety that is attached to one of its $\mathrm{N}$-type glycans. ${ }^{67}$ We now show that the anti-inflammatory effects of ES-62, in both the CIA model and human patient samples, are dependent on the PC moiety and indeed, can be mimicked by synthetic PC-containing molecules. Identification of this key structural component of ES-62 as an anti-inflammatory agent provides the first step towards addressing the development of ES-62-based drugs for treating autoimmunity. This is a particularly attractive idea as the evolution of the host-parasite relationship has produced in ES62 , a molecule that is immunomodulatory but at the same time "safe", in leaving defences against infection, intact.

\section{METHODS AND MATERIALS}

\section{Animals}

Male DBA/1 mice obtained from Harlan Olac (Bicester, UK) were used at 8-10 weeks of age and maintained at the Joint Animal Facilities, University of Glasgow. All animals were cared for in accordance to the Home Office, UK animal guidelines.

\section{ES-62 and phosphorylcholine conjugated to ovalbumin preparations}

Purified, endotoxin-free ES-62 from the rodent filarial nematode $A$. viteae was prepared as described previously. ${ }^{8}$ Sequence comparison of 
human filarial homologues (Brugia malayi) of ES-62 reveals $>70 \%$ homology at the amino acid level and the presence of conserved sites for post-translational modification with PC-Nglycans. ${ }^{9} \mathrm{PC}$ conjugated to ovalbumin (OVA-PC) and OVA were synthesised as described previously. ${ }^{10}$ Recombinant ES-62 lacking PC (rES-62) was prepared following expression in Pichia pastoris as described previously. ${ }^{11}$ Absence of endotoxin from ES-62, rES-62 and PC-materials was confirmed using an Endosafe Kit (Charles River Laboratories, Kent, UK).

\section{Induction and assessment of collagen-induced arthritis}

Male DBA/1 mice received $200 \mu \mathrm{g}$ of bovine type II collagen (CII, Sigma, Poole, UK) in Freund's complete adjuvant (Difco, Detroit, MI, USA) by intradermal injection (day 0). Collagen (200 $\mu \mathrm{g}$ in phosphate-buffered saline (PBS)) was given again on day 21 by intraperitoneal injection. Mice were monitored daily for signs of arthritis for which severity scores were derived as follows: $0=$ normal, $1=$ erythema, 2 = erythema plus swelling, $3=$ extension/loss function, and total score $=$ sum of four limbs. For the prophylactic protocol, mice were treated with $2 \mu \mathrm{g}$ ES-62 or other molecules subcutaneously on day -2 , day 0 and day 21 . For the therapeutic studies, mice were treated daily with ES-62 or other molecules, $2 \mu$ g subcutaneously for 14 days commencing 1 day after CIA was clinically detectable. Control mice received $\mathrm{PBS}$ alone at the same time points.

\section{Ex vivo culture conditions}

Draining lymph node cells and splenocytes were cultured at $2 \times 10^{6} / \mathrm{ml}$ for up to $96 \mathrm{~h}$ in RPMI medium, supplemented as described previously. ${ }^{10}$ Cells were stimulated with CII $(50 \mu \mathrm{g} /$ $\mathrm{ml}), \mathrm{F}\left(\mathrm{ab} \mathrm{b}_{2}\right.$ fragments of anti-immunoglobulin antibodies $(50 \mu \mathrm{g} / \mathrm{ml})$ or concanavalin A $(5 \mu \mathrm{g} / \mathrm{ml})$. For proliferation assays, $\left[{ }^{3} \mathrm{H}\right]$-thymidine (Amersham, Little Chalfont, UK) was added for the last $18 \mathrm{~h}$ of culture while supernatants from parallel triplicate cultures were stored at $-70^{\circ} \mathrm{C}$ until estimation of cytokine content by ELISA or Luminex. ${ }^{12} 13$

\section{Human studies}

Samples were derived with approval from the Glasgow Royal Infirmary Ethical Committee. Patients with RA fulfilled American College of Rheumatology diagnostic criteria. Primary synovial membrane cultures were obtained as previously described by collagenase (Worthington, Lakewood, NJ, USA)/DNAse (Sigma-Aldrich, Gillingham, Dorset, UK) digestion of synovial membrane samples obtained at knee arthroplasty. ${ }^{14}$ Peripheral blood and synovial fluid were obtained from patients with RA and mononuclear cells prepared in Lymphoprep by density gradient centrifugation. Synovial membrane or synovial fluid cultures were at $2 \times 10^{6} / \mathrm{ml}$ in the presence or absence of the indicated stimuli for $48 \mathrm{~h}$.

\section{Cytokine and immunoglobulin analysis}

Tumour necrosis factor (TNF)- $\alpha$, interferon (IFN)- $\gamma$, interleukin (IL)-5, IL-6 and IL-10 were detected by OptEIA ELISA kits and IL-12p40/p70 and transforming growth factor (TGF)- $\beta$ were analysed using antibody pairs (all BD PharMingen, San Diego, CA, USA) or Luminex assays according to the manufacturer's instructions. Detection limits were as follows: IL-5, IL-6 and TNF- $\alpha$ all at $10 \mathrm{pg} / \mathrm{ml}$; IL-10, IL-12 and IFN- $\gamma$ at $20 \mathrm{pg} / \mathrm{ml}$ and TGF- $\beta$ at $30 \mathrm{pg} / \mathrm{ml}$. TGF- $\beta$ samples were acidified to release the biologically active form prior to measurement as described by BD PharMingen. Human TNF- $\alpha$ and IL-6 (Biosource, Nivelles, Belgium) were assayed by ELISA with limits of detection of
$30 \mathrm{pg} / \mathrm{ml}$. For Luminex analysis, Bioplex Manager software with five parametric curve fitting was used for data analysis (BioRad, Hemel Hempstead, UK). Unlike ELISA that provides a single OD measurement per well, Luminex provides a value based on the mean of at least 100 measurements per sample. Anticollagen II antibody titres of individual sera were detected by ELISA: serum samples were titrated on ELISA plates coated with $20 \mu \mathrm{g} / \mathrm{ml}$ type II collagen and then developed with biotinconjugated anti-mouse IgG1 or IgG2a (PharMingen), followed by conjugated avidin peroxidase (Sigma) and developed with tetramethylbenzidine substrate (Kirkegaard and Perry, Gaithersburg, MD).

\section{RESULTS}

\section{The role of phosphorylcholine in the anti-inflammatory effects of} ES-62 in the collagen-induced arthritis model

We have previously shown that while ES-62 does not reduce the incidence of CIA, it suppresses the severity of disease. ${ }^{5}$ To investigate whether the PC moiety is responsible for this, rES-62 lacking PC was used to treat mice in the prophylactic and therapeutic treatment regimens. In contrast to native ES-62, prophylactic treatment with $\mathrm{rES}-62$ did not significantly inhibit the severity of CIA (fig 1A). Nevertheless, like ES-62, rES-62 treatment significantly reduced levels of CII-specific IgG2a, without a compensatory modulation of CII-specific IgG1, IgG3 or IgM (fig $1 \mathrm{~B}$ and results not shown). Therapeutic treatment of mice with rES-62 after the onset of inflammation did not significantly modulate the incidence or severity of arthritis, or the profiles of collagen-specific IgG1, IgG2a, IgG3 or IgM, relative to PBS-treated mice (results not shown).

These results support the proposal that PC may mediate the effects of ES-62 observed in the CIA model. However, rES-62 is not simply a PC-free copy of native ES-62: it also contains higher levels of mannose and has been shown to exhibit differential secondary and tertiary structure. ${ }^{11}$ To investigate whether PC, independently of the rest of ES-62, could inhibit inflammation exhibited in the CIA model, we treated mice with PC conjugated to an irrelevant protein, ovalbumin (OVA-PC). As a negative control for OVA-PC, a group of mice were treated with sham-conjugated ovalbumin (OVA). Prophylactic treatment of mice with OVA-PC significantly reduced the severity of inflammation, when compared with the OVA treatment group (fig 1C), suggesting that PC was independently capable of antiinflammatory action in this arthritis model. However, serum samples from OVA and OVA-PC-treated CIA model mice exhibited no significant differences in their levels of CII-specific IgG1, IgG2a and IgG3, indicating that, unlike ES-62, PC did not specifically modulate antigen-specific IgG (results not shown). Therapeutic treatment of mice with OVA-PC, but not OVA, after the onset of clinically detectable arthritis also prevented progression of the inflammation (fig 1D). Again, analysis of serum immunoglobulins revealed no significant differences in the levels of CII-specific IgG1, IgG2a, IgG3 and IgM between OVA- and OVA-PC-treated mice (results not shown).

\section{ES-62 does not modulate the serum factor profile of collagen- induced arthritis model mice}

Serum samples from the PBS group mice (day 33) were found to contain measurable quantities of the pro-inflammatory cytokines, IL-1 $\alpha$, IL-12 and IL-2. Similarly, KC, MIP- $1 \alpha$, IP-10 and MIG, inflammatory chemokines that recruit neutrophils ${ }^{15} 16$ and are important markers of TNF- $\alpha^{17}$ and IFN- $\gamma$-mediated inflammation $^{18} 19$ were also detected, along with fibroblast growth 
Figure 1 OVA-PC, but not rES-62, reduces severity of collagen-induced arthritis. $(A-C, E, F) D B A / 1$ mice were immunised on days 0 and 21 with collagen, and were treated with ES-62 $(n=9), r E S-62(n=9)$, OVA-PC $(n=9)$, OVA $(n=9)$ or PBS $(n=9)$ on days $-2,0$ and 21. Clinical score was monitored daily. For mean articular index $(A, C)$, data are expressed as mean (SEM).

$* * * p<0.001$ : ES-62 compared with rES62 (and PBS) and ${ }^{* *} p<0.01$ OVA compared with OVA-PC. (B) Collagenspecific IgG2a levels in serum samples from PBS-, ES-62- and rES-62-treated mice were measured by ELISA. Data are expressed as mean concentration $(\mathrm{pg} / \mathrm{ml})$ (SEM) from individual mice ( $\mathrm{n}=9 /$ group), ${ }^{*} \mathrm{p}<0.05$ rES-62 or ES-62 versus PBS. (D) $\mathrm{DBA} / 1$ mice were treated daily, 1 day after detectable clinical collagen-induced arthritis with OVA-PC $(n=5)$ or OVA $(n=6)$. Mean articular index was monitored daily and data are expressed as mean (SEM) and ${ }^{* *} p<0.001$ for OVA compared with OVA-PC. Draining lymph nodes and spleens from the prophylactic protocols were obtained for each treatment group after they were killed and cellular proliferative responses assessed by $\left[{ }^{3} \mathrm{H}\right]$ thymidine uptake. Spleen cells (E) were stimulated with media or $\mathrm{F}\left(\mathrm{ab}^{\prime}\right)_{2}$ fragments of anti-immunoglobulin antibodies $(50 \mu \mathrm{g} / \mathrm{ml})$ for $72 \mathrm{~h}$ and draining lymph node cells (F) were stimulated with media or collagen (F; CIl, $50 \mu \mathrm{g} / \mathrm{ml}$ ) for $72 \mathrm{~h}$. Data are plotted as the mean values from the indicated number of individual mice (of triplicate determinations) from each treatment group. (E) ${ }^{*} \mathrm{p}<0.05$ for $\mathrm{rES}-62$ versus PBS and ${ }^{* * *} p<0.001$ for ES-62 versus PBS, and $(F){ }^{* * *} p<0.001$ for ES-62 or OVA-PC versus PBS and OVA (Student's t-test). All data are representative of at least three independent experiments. PBS, phosphate-buffered saline; PC, phosphorylcholine; OVA-PC, PCovalbumin conjugate.
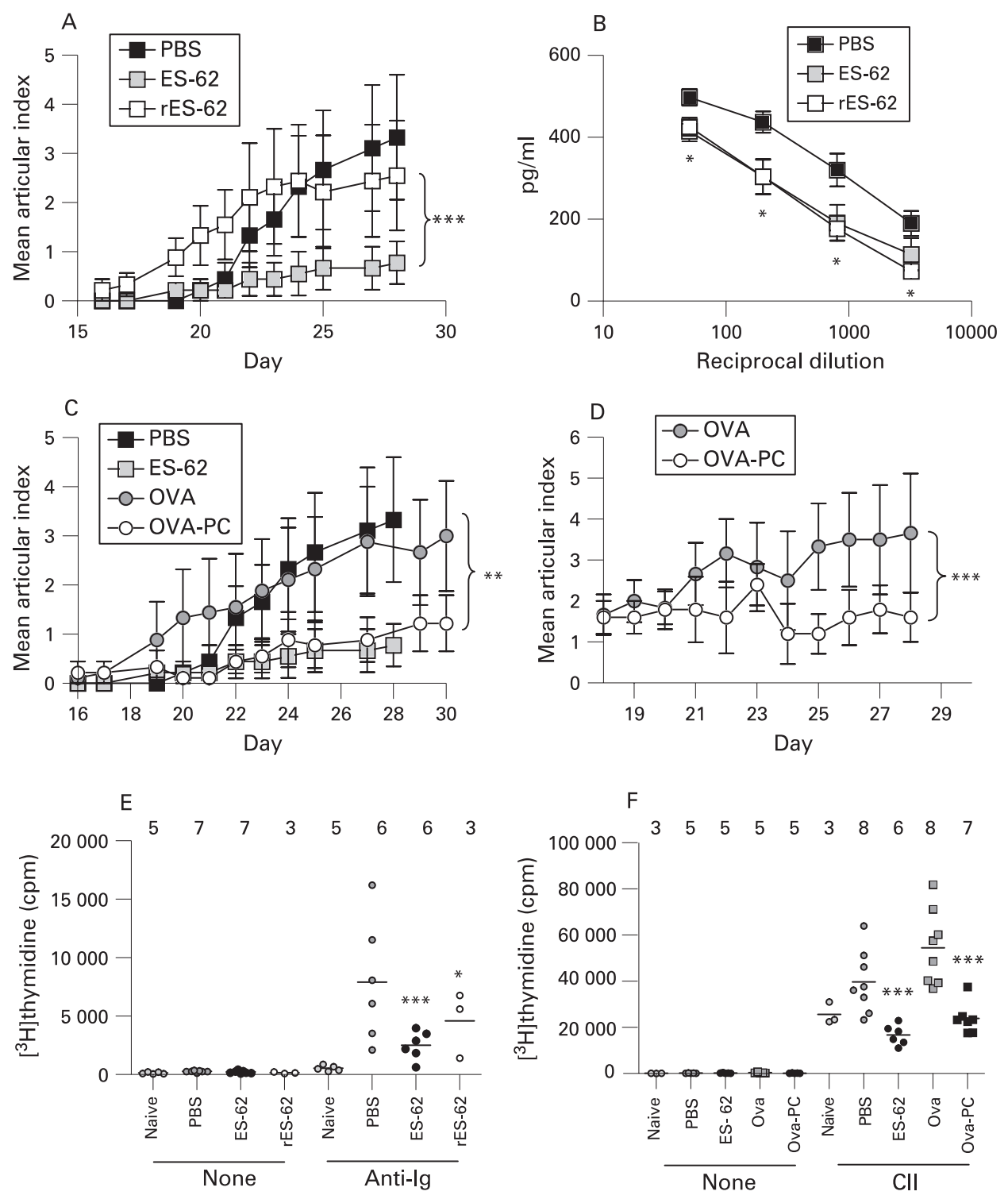

factor-basic (FGF-2) a potent stimulator of cellular proliferation and angiogenesis. ${ }^{20}$ Prophylactic or therapeutic treatment of mice with ES-62 or OVA-PC, however, did not induce any significant effects on the serum levels of these inflammatory mediators (results not shown).

\section{Investigation of the role of phosphorylcholine in ES-62-mediated modulation of immune cell responses}

We have previously demonstrated that the anti-inflammatory actions of ES-62 in CIA correlate with suppression of Th1 responses in draining lymph node cells (DLN) challenged with antigen (CII), but not mitogen (concanavalin A), ex vivo. ${ }^{5}$ To further address the role of PC, we assessed the effects of ES-62, rES-62 and OVA-PC on immune system cell number and subset distribution following induction of CIA in vivo. These studies showed that none of the treatments significantly altered the mean number of total lymph node or splenic cell populations after induction of CIA. Moreover, relative proportions of B220+
$\mathrm{B}$ cells, $\mathrm{CD}^{+} \mathrm{T}$ helper cells and $\mathrm{CD}^{+}$cytotoxic $\mathrm{T}$ cells were not modulated by any of these treatments (results not shown). Consistent with this, in subsequent ex vivo cultures, such DLN and splenic cells were as responsive to stimulation with the mitogen, concanavalin A as those from PBS or OVA-treated mice (results not shown). In contrast, ES-62- and rES-62-treated splenic cells were hyporesponsive to stimulation via the BCR (anti-immunoglobulin). Moreover, ES-62- and OVA-PC-, but not rES-62- or OVA-treated cells were refractory to antigen (CII) stimulation of $\mathrm{T}$ cells, results consistent with our previous findings on the in vivo action of ES-62 in normal and CIA mice $^{578}$ (fig 1E,F and results not shown).

Reflecting these findings, the ex vivo responses of DLN and splenic cultures from CIA mice to concanavalin A in producing a wide range (TNF- $\alpha$, IL-12, IL-6, IL-2, IFN- $\gamma$, IL-17, IL-5, IL-13 and granulocyte-macrophage colony-stimulating factor) of cytokines were not modulated by previous in vivo exposure to ES-62, rES-62 or OVA-PC (results not shown). However, ES-62 

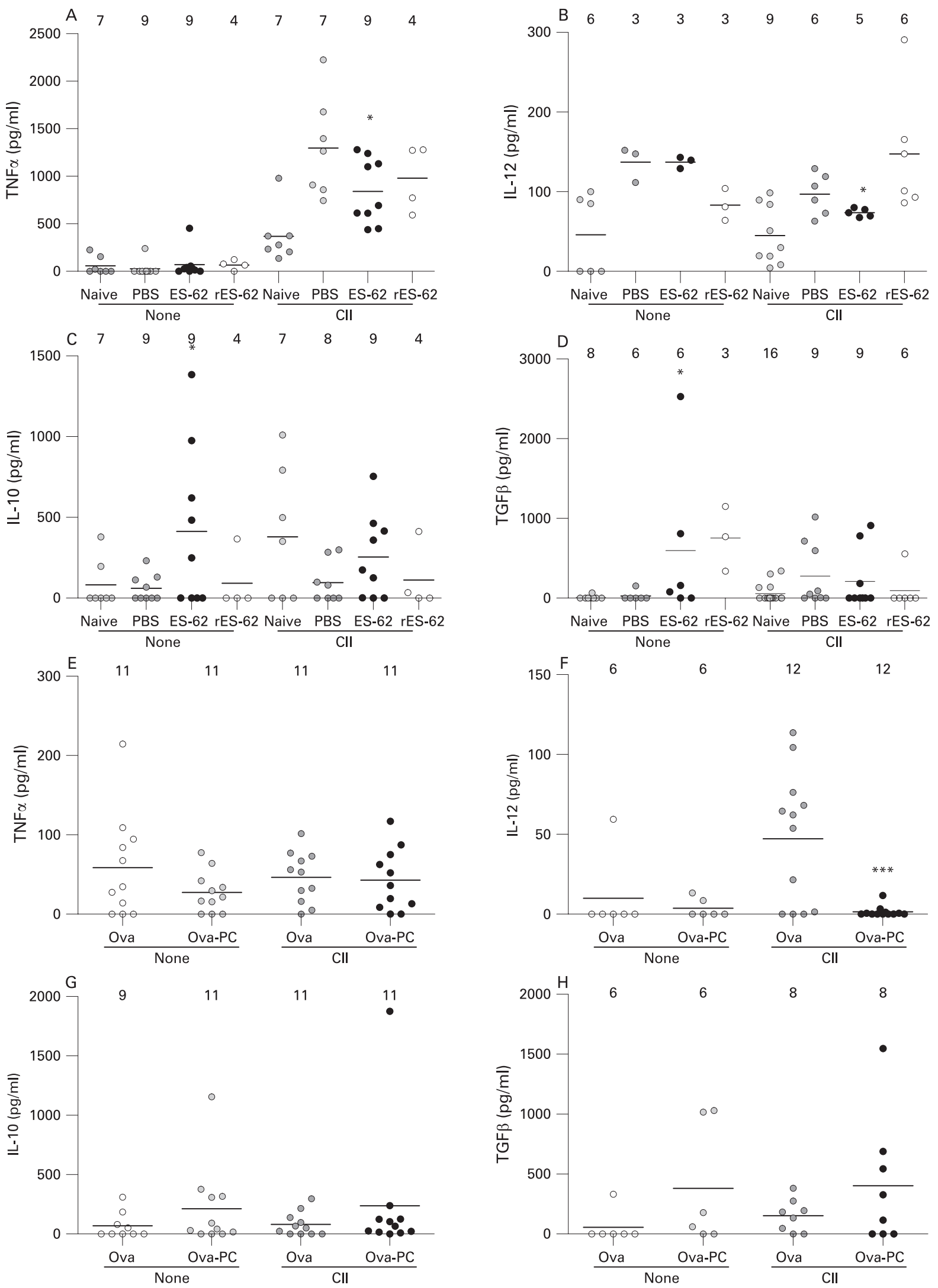

Figure 2 Comparison of ex vivo collagen-stimulated cytokine responses from CIA model mice treated with ES-62, 0VA-PC and rES-62. DBA/1 mice were immunised on days 0 and 21 with collagen, and were treated with ES-62, rES-62, OVA-PC, OVA or PBS as indicated on days -2, 0 and 21. Splenic cells were obtained from naive mice and from each treatment group at day 33 and stimulated with media or collagen (Cll, $50 \mu \mathrm{g} / \mathrm{ml}$ ) for $96 \mathrm{~h}$ and cytokine secretion assessed by ELISA. Data are plotted as the mean of triplicate samples from the indicated number of individual mice in each of the treatment groups; the bar represents the mean of mean values for the group. Data are representative of three independent experiments. ${ }^{*} p<0.05$ for ES-62 versus PBS and ${ }^{* * *} p<0.001$ for OVA-PC versus OVA (Student's t-test). CIA, collagen-induced arthritis; TNF, tumour necrosis factor; PBS, phosphate-buffered saline; TGF, transforming growth factor; IL, interleukin; PC, phosphorylcholine; OVA-PC, PC-ovalbumin conjugate. 

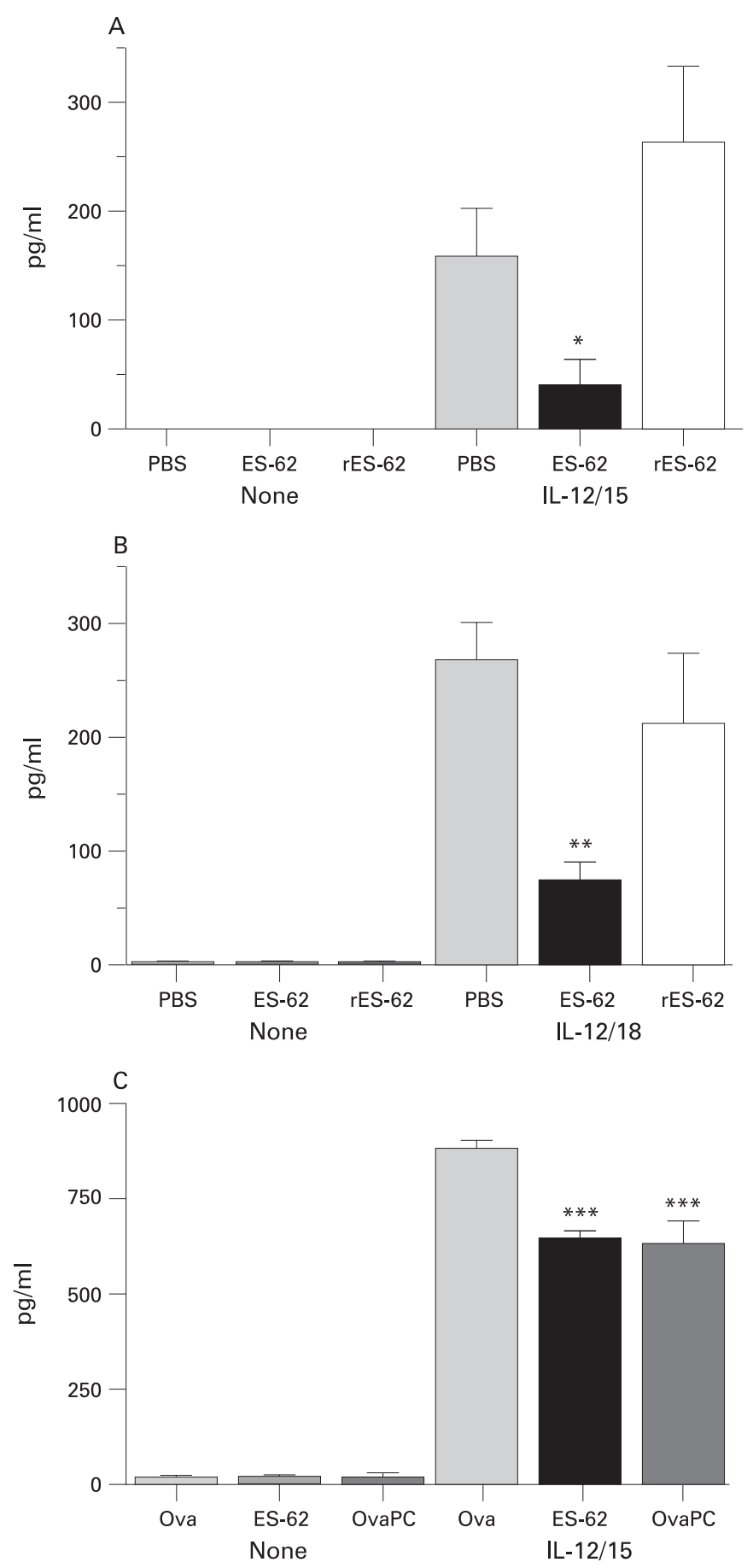

Figure 3 The role of PC in ES-62-mediated inhibition of interferon- $\gamma$ responses by human patient samples with RA. Mononuclear cells from peripheral blood $(A)$ and synovial membrane cultures $(B, C)$ of patients with RA were stimulated overnight with media, IL-12 (2 ng/ml), IL-15 (50 ng/ml) or IL-18 (50 ng/ml) following pre-exposure to PBS, ES-62, rES-62 (both $2 \mu \mathrm{g} / \mathrm{ml}$ ), OVA or OVA-PC (both $10 \mu \mathrm{g} / \mathrm{ml}$ ) for $4 \mathrm{~h}$ as indicated and culture supernatants analysed for interferon- $\gamma$ by ELISA. Data are presented as mean values of triplicate cultures (SD) from individual patients and are representative of data from synovial membrane cultures from eight patients with RA and peripheral blood from five patients with RA. (A) ${ }^{*} p<0.05$ for ES-62 versus PBS and $r E S$ 62; (B) ${ }^{* *} p<0.01$ for ES-62 versus PBS and rES-62; and (C) ${ }^{* * *} p<0.001$ for ES-62 and OVA-PC versus OVA (Student's t-test). PBS, phosphate-buffered saline; PC, phosphorylcholine; OVA-PC, $\mathrm{PC}$-ovalbumin conjugate; IL, interleukin; RA, rheumatoid arthritis. significantly inhibited collagen-specific TNF- $\alpha$ and IL-12 responses but this was not the case with rES-62 (fig 2A,B). Moreover, exposure to OVA-PC in vivo also led to significantly reduced antigen-specific IL-12, but this was not noted with TNF- $\alpha$, responses (fig $2 \mathrm{E}, \mathrm{F}$ ), suggesting that PC played a key role in the former immunomodulatory effect of ES-62. Interestingly, pre-exposure to ES-62, but not rES-62, in vivo leads to enhanced spontaneous production of the regulatory cytokines IL-10 and TGF- $\beta$ by splenocytes from CIA mice (fig 2C,D). However, although some increases in spontaneous and CII-induced IL-10 and TGF- $\beta$ responses were observed in cells derived from OVA$\mathrm{PC}$ relative to OVA-treated CIA mice (fig $2 \mathrm{G}, \mathrm{H}$ ), these were generally not significant.

\section{Role of phosphorylcholine in the therapeutic potential of ES-62 in rheumatoid arthritis in humans}

Our previous studies showed that in addition to suppressing CIA in mice, ES-62 exhibited anti-inflammatory activity in peripheral blood mononuclear cell and synovial fluid and membrane cultures from human patients with RA. In these studies, we showed that ES-62 could inhibit LPS-induced proinflammatory cytokine production from peripheral blood mononuclear cells and synovial cells and also from macrophages activated by $\mathrm{T}$ cells in a cell contact-dependent manner. ${ }^{5} \mathrm{We}$ have now extended these studies to show that peripheral blood mononuclear cells and synovial fluid and membrane cultures from patients with RA, stimulated with the cytokines IL-12, IL15 and IL-18, to mimic the pro-inflammatory milieu of the joint, ${ }^{514} 21$ exhibit reduced capacity to secrete the signature Th1 cytokine, IFN- $\gamma$ when pre-exposed to ES-62 or OVA-PC but not rES-62 or OVA (fig 3, and results not shown).

\section{DISCUSSION}

We have previously established that ES-62 can inhibit Th1-type immune responses in the CIA model of inflammatory autoimmune RA and in human RA patient blood and synovial samples. ${ }^{5}$ Here, we demonstrate that the anti-inflammatory action of ES-62 is lost when a PC-deficient recombinant homologue of ES-62 (rES-62) is employed. This potentially important role of PC in ES-62-mediated inhibition of rheumatological inflammation is corroborated by the finding that OVA$\mathrm{PC}$, is capable of mimicking the anti-inflammatory actions of ES-62 in both the CIA model and in human patient samples.

Ultimately, inflammation and pathology in CIA occurs as a result of developing a chronic collagen (CII)-specific, Th1-type immune response. Previously, it was demonstrated that ES-62mediated inhibition of inflammation in the CIA model was associated with reduced antigen-specific IgG2a, a Th1-promoting antibody subclass, with no modulation of IgG1, IgG3 and IgM levels. Interestingly, the anti-inflammatory action of OVAPC on CIA model mice was not associated with significant modulation of the level or nature of the serum IgG response, indicating that the inhibitory action of ES-62 on antigen-specific IgG2a was PC-independent. Furthermore, despite lacking antiinflammatory action in the CIA model, treatment of mice with PC-deficient rES-62 induced inhibition of antigen-specific IgG2a production, similar to that induced by treatment with parasitederived ES-62. Thus, inhibition of collagen-specific IgG2a by native and $\mathrm{rES}-62$ indicates that this effect was mediated by a non-PC, ES-62-specific, component(s) and presumably reflects the ability of both ES-62 and rES-62 to inhibit BCR-mediated B cell proliferation. The apparent inconsistency in anti-inflammatory action and IgG2a inhibition indicated that the serum 
antibody profile did not reflect the inflammatory status, in terms of cytokine production and arthritic score, of the mice in the CIA model. Although at first sight this was surprising given that IgG2a levels are generally considered to correspond to inflammation severity in CIA, some other studies have argued against a correlation between IgG2a levels and CIA incidence in mice and have demonstrated maintenance of a Th1 immune response, despite absence of an IgG2a antibody response. ${ }^{22}{ }^{23} \mathrm{In}$ relation to this, we also failed to find a correlation between the antigen-specific splenic IL-12 response and serum IgG2a levels with respect to the various stimulants.

Although TNF- $\alpha$ was not detected in serum samples from CIA model mice, it is possible that TNF- $\alpha$ was still acting locally, at the inflammatory site for example, inducing production of KC and MIP- $1 \alpha$ at this location, but not circulating in the bloodstream. Consistent with this, DLN and splenocytes from CIA mice exposed to ES-62 but not rES-62, in vivo showed reduced capacity to generate TNF- $\alpha$ in recall responses to collagen ex vivo. Similarly, although serum IL-10 levels were not induced by ES-62 treatment, ex vivo DLN and splenocyte cultures from ES-62-treated mice exhibited enhanced spontaneous IL-10 responses suggesting that this cytokine, which has been demonstrated to act in an anti-inflammatory manner on synovial cells, ${ }^{24}$ particularly in terms of regulating TNF- $\alpha$ in RA, ${ }^{25}$ may play some part in the ES-62-mediated inhibition of inflammation in CIA. Similarly, although a role for TGF- $\beta$ was not implicated by serum analysis, spontaneous production of this regulatory cytokine was found in ES-62treated DLN responses. Surprisingly, none of these effects on cytokines was observed at a statistically significant level when employing OVA-PC in spite of its anti-inflammatory activity, but as mentioned above a similar failure to modulate the IgG anti-CII response was also observed.

In conclusion, our findings suggest that PC largely mimics the anti-inflammatory action of ES-62 in CIA, in particular being associated with the reduction of antigen-specific Th1 cytokine production. However, not all of the effects of ES-62 on this model required PC as the ES-62-mediated inhibition of CIIIgG2a production was $\mathrm{PC}$-independent, suggesting that this particular action on antibody levels is not directly associated with, or necessary for, reduction of inflammation. PC-containing homologues of $A$ viteae ES-62 are produced by human filarial nematode parasites. Thus, the reduced incidence of inflammatory autoimmune diseases, such as RA, in countries where filariasis is endemic could be attributed to the anti-inflammatory action of these molecules.

Funding: This work was supported by the Wellcome Trust (DEK \& CNS are PhD training fellows), the BBSRC and Synergy/Proof of Concept awards. TT is an Oliver Bird $\mathrm{PhD}$ training fellow.

Competing interests: None.

\section{REFERENCES}

1. Kalla AA, Tikly M. Rheumatoid arthritis in the developing world. Best Pract Res Clin Rheumatol 2003;17:863-75.
2. Cooke A, Zaccone P, Raine T, Phillips JM, Dunne DW. Infection and autoimmunity: are we winning the war, only to lose the peace? Trends Parasitol 2004;20:316-21.

3. Harnett W, Harnett MM, Byron 0. Structural/functional aspects of ES-62-a secreted immunomodulatory phosphorylcholine-containing filarial nematode glycoprotein. Curr Protein Pept Sci 2003;4:59-71.

4. Harnett W, McInnes IB, Harnett MM. ES-62, a filarial nematode-derived immunomodulator with anti-inflammatory potential. Immunol Lett 2004:94:27-33.

5. McInnes IB, Leung BP, Harnett M, Gracie JA, Liew FY, Harnett W. A novel therapeutic approach targeting articular inflammation using the filarial nematodederived phosphorylcholine-containing glycoprotein ES-62. J Immunol 2003;171:212733.

6. Harnett W, Harnett MM. Phosphorylcholine: friend or foe of the immune system? Immunol Today 1999;20:125-9

7. Harnett W, Deehan MR, Houston KM, Harnett MM. Immunomodulatory properties of a phosphorylcholine-containing secreted filarial glycoprotein. Parasite Immunol 1999;21:601-8

8. Wilson EH, Deehan MR, Katz E, Brown KS, Houston KM, O'Grady J, et al. Hyporesponsiveness of murine B lymphocytes exposed to the filarial nematode secreted product ES-62 in vivo. Immunology 2003;109:238-45.

9. Goodridge HS, Stepek G, Harnett W, Harnett MM. Signalling mechanisms underlying subversion of the immune response by the filarial nematode secreted product ES-62. Immunology 2005;115:296-304.

10. Goodridge HS, Marshall FA, Else KJ, Houston KM, Egan C, Al-Riyami L, et al. Immunomodulation via novel use of TLR4 by the filarial nematode phosphorylcholinecontaining secreted product, ES-62. J Immunol 2005;174:284-93.

11. Egan CA, Houston KM, Alcocer MJ, Solovyova A, Tate R, Lochnit G, et al. Lack of immunological cross-reactivity between parasite-derived and recombinant forms of ES-62, a secreted protein of Acanthocheilonema viteae. Parasitology 2006;132:26374.

12. Marshall FA, Grierson AM, Garside P, Harnett W, Harnett MM. ES-62, an immunomodulator secreted by filarial nematodes, suppresses clonal expansion and modifies effector function of heterologous antigen-specific T cells in vivo. J Immunol 2005; 175:5817-26.

13. Kean DE, Goodridge HS, McGuiness S, Harnett MM, Alcocer MJC, Harnett W. Differential polarization of immune responses by plant $2 \mathrm{~S}$ seed albumins, Ber e 1 , and SFA81 Journal of Immunology 2006;177:1561-6.

14. Mclnnes IB, Leung BP, Sturrock RD, Field M, Liew FY. Interleukin-15 mediates T cell-dependent regulation of tumor necrosis factor-alpha production in rheumatoid arthritis. Nat Med 1997;3:189-95.

15. Sherry B, Horii Y, Manogue KR, Widmer U, Cerami A. Macrophage inflammatory proteins 1 and 2: an overview. Cytokines 1992;4:117-30.

16. Lira SA, Zalamea P, Heinrich JN, Fuentes ME, Carrasco D, Lewin AC, et al Expression of the chemokine N51/KC in the thymus and epidermis of transgenic mice results in marked infiltration of a single class of inflammatory cells. J Exp Med 1994: 180:2039-48.

17. Ohmori Y, Wyner L, Narumi S, Armstrong D, Stoler M, Hamilton TA. Tumor necrosis factor-alpha induces cell type and tissue-specific expression of chemoattractant cytokines in vivo. Am J Pathol 1993:142:861-70.

18. Ren X, Kennedy A, Colletti LM. CXC chemokine expression after stimulation with interferon-gamma in primary rat hepatocytes in culture. Shock 2002;17:513-20.

19. Gasperini S, Marchi M, Calzetti F, Laudanna C, Vicentini L, Olsen H, et al. Gene expression and production of the monokine induced by IFN-gamma (MIG), IFNinducible T cell alpha chemoattractant (I-TAC), and IFN-gamma-inducible protein-10 (IP-10) chemokines by human neutrophils. J Immunol 1999;162:4928-37.

20. Presta M, Dell'Era P, Mitola S, Moroni E, Ronca R, Rusnati M. Fibroblast growth factor/fibroblast growth factor receptor system in angiogenesis. Cytokine Growth Factor Rev 2005;16:159-78.

21. McInnes IB, Leung BP, Liew FY. Cell-cell interactions in synovitis. Interactions between T lymphocytes and synovial cells. Arthritis Res 2000;2:374-8.

22. Bouvet JP, Couderc J, Bouthillier Y, Franc B, Decreusefond C, Mouton D. Collageninduced arthritis in Biozzi mice. Joint involvement is not correlated with collagen II IgG2a autoantibodies nor restricted to only $\mathrm{H}-2 \mathrm{q}$ and $\mathrm{H}-2 \mathrm{r}$. J Immunol 1989;143:1537-42.

23. Satoskar AR, Stamm LM, Zhang X, Okano M, David JR, Terhorst C, et al. NK celldeficient mice develop a Th1-like response but fail to mount an efficient antigenspecific IgG2a antibody response. J Immunol 1999;163:5298-302.

24. Feldmann M, Brennan FM, Maini RN. Role of cytokines in rheumatoid arthritis. Annu Rev Immunol 1996;14:397-440.

25. Katsikis PD, Chu CQ, Brennan FM, Maini RN, Feldmann M. Immunoregulatory role of interleukin 10 in rheumatoid arthritis. J Exp Med 1994:179:1517-27. 